\title{
Nicht nur Fokuspartikel: Geschichte und Varianz von nur im Deutschen
}

\section{Einführung: Verfestigung und Pragmatisierung}

\subsection{Ni waere und die einzige positive Ausnahme zu einem negativen Urteil}

Das neuhochdeutsche Signans nur geht auf die Verfestigung einer verbalen Konstruktion ni waere /ne wâre/neuuare (fortan: ni waere), „wäre nicht“, im Frühmittelhochdeutschen zurück. Diese Wendung bestand aus der Negation ne/ni, „nicht“ und einer Konjunktivform des Verbs sein. Letztere lässt sich durchaus vergleichen mit dem sei in der ebenfalls verfestigten Konstruktion es sei denn, die die ursprüngliche Rolle von ni waere im Wesentlichen übernommen hat. Als sie sich verfestigte, diente die Konstruktion ni waere dazu, eine Ausnahme zu der unmittelbar vorher geäußerten Assertion auszudrücken. In dieser Hinsicht kann ni waere eine konnektorähnliche Funktion zugesprochen werden. Außerdem schien der Gebrauch von ni waere, zumindest in den ersten Belegen, auf Kontexte beschränkt zu sein, in denen es darum ging, positive Ausnahmen zu einem vorherigen negativen Urteil zu nennen.

(1) in eius conuersione negesihest du nieth anderes neuuare daz du nu sihest in dinen conuenticulis. [Willeram, zit. nach DWB XIII: 999]

in eius conversione ne-ge-sihest $d u$ nieht anderes in seiner ${ }^{1}$ Bekehrung NEG-PERF-siehst $d u$ nichts anderes $\begin{array}{llllllll}\text { neuuare } & \text { daz } & d u & n u & \text { sihest in dinen conventiculis } \\ \text { neuuare } & \text { was } & d u & \text { nun } & \text { siehst in deinen } & \text { Versammlungen }\end{array}$ „Bei seiner Bekehrung wirst du nichts anderes betrachten, als das, was du nun in deinen Kirchenversammlungen siehst.“

1 Es geht um die für unausweislich gehaltene kollektive Bekehrung des gesamten Volkes Israels zum Christentum. Der Adressat ist selber schon Mitglied einer kleinen Kirche. 
Diese Rolle wurde im Laufe der mhd. Zeit von denn bzw. es sei denn übernommen:

(2) ich lasse dich nit, nur du gesegnest mich. (4. Bibelübersetzung, 1 Mos. 32, 26; du segnest mich denn, Luther) [DWB XIII: 999]

(3) Keiner kumpt zu dem vatter, nur durch mich. Joh. 14, 6 (denn durch mich, Luther) [zit. DWB XIII: 999]

Diese Beschreibung des Etymons von nur weist sofort auf eine mögliche Paraphrase der sog. „exklusiven Fokuspartikel“ im heutigen Deutsch: Wird in einer Aussage $\mathbf{p}$ eine fokussierte Konstituente e mit nur markiert, so bedeutet dies, dass e innerhalb eines Paradigmas die einzige Entität denotiert, für welche $\mathbf{p}$ nicht falsch ist.

(4) ez geschiht niur was got wil. [Teichard, zit. DWB XIII: 1000]

Es besteht also eine unübersehbare Ähnlichkeit zwischen der Bedeutung des ehemaligen Junktors und dem heutigen Gebrauch als Fokuspartikel. Nur wäre demnach ein interessanter Fall von Pragma- oder Grammatikalisierung ${ }^{2}$ mit einer deutlichen formalen Reduzierung, einer Entwicklung des syntaktischen Status, aber zugleich einer unübersehbaren semantischen Beständigkeit. In Anlehnung an Martine Dalmas' fortwährendes Interesse für argumentative Marker und Phänomene der Lexikalisierung, der Grammatikalisierung und der Pragmatisierung wollen wir uns im Folgenden dieses nur aufs Korn nehmen. Dabei soll es um folgende Fragen gehen: Lassen sich die heutigen Gebrauchsweisen von nur überhaupt auf diesen Grundwert des Etymons zurückführen, und wenn ja, inwieweit? Kann dieser Zusammenhang sogar zu einer semantischen Reduktion im Sinne des Bedeutungsminimalismus führen? (siehe Posner 1979) Oder im Falle der Polysemie, wie lassen sich die verschiedenen Bedeutungen aus dem Urwert diachron ableiten? Wir wollen uns dabei auf das Dissertationskorpus von Modicom

\footnotetext{
2 Auf die Debatte zum Verhältnis zwischen Grammatikalisierung und Pragmatisierung wollen wir nicht näher eingehen. Zur Grammatikalisierung sei nur auf Meillet (1912) und Lehmann (2015 [1982]) hingewiesen. Für Pragmatisierung siehe etwa Dostie (2004). Für das Verhältnis zwischen beiden siehe Badiou-Monferran/Buchi (2012) sowie auch Traugott (1989), die ein alternatives Modell von Grammatikalisierung vorschlägt, in dem der Begriff Pragmatisierung überflüssig sein soll.
} 
$(2016)^{3}$ stützen sowie auf eine Reihe von opportunistisch gesammelten Beispielen, die allein aufgrund der semantischen Bandbreite selegiert wurden. Einen Anspruch auf Repräsentativität erheben wir also nicht, da es uns im Moment eher um die Messung der diskursiven Variationsbreite überhaupt geht sowie um die Möglichkeit ihrer semantischen Reduktion.

\subsection{Einverleibung der negativen Orientierung: Nur als exklusive Fokuspartikel}

Fangen wir an mit der Fokuspartikel, zunächst in ihrem exklusiven Gebrauch. Eigentlich lassen sich die Deutungen der „exklusiven Fokuspartikel“ nur in zwei Gruppen einteilen: den skalaren und den nicht-skalaren (König 1991).

nicht-skalar:

(5) Den Rabbi verdroß es zuweilen, daß Naftali mit seinen Scherzworten nie innehielt und niemand schonte. Einmal verlangte er ihm ab, er solle ein Jahr lang die Späße unterlassen. Aber Naftali wollte sich dazu nur unter der Bedingung verstehen, daß der Rabbi versprach, nicht, wie er oft tat, länger als eine Stunde im stillen Gebet zu stehen und dadurch den Vorsänger mitsamt den Betern aufzuhalten. (Martin Buber, Gog und Magog: 70)

skalar:

(6) Laut einer Infratest-dimap-Umfrage glauben nur 18 Prozent der Deutschen, Gabriel könnte Merkel 2017 schlagen. 40 Prozent meinen, Außenminister Frank-Walter Steinmeier (SPD) wäre der aussichtsreichste Herausforderer. (Süddeutsche Zeitung, 8. April 2015)

Ob diese strenge Dichotomie wirklich haltbar ist, darf bezweifelt werden. Folgendes Beispiel etwa ist zumindest grenzwertig:

(7) Es ist leider so, das ist unser Schicksal, dass im Moment nur die CDU für uns als Koalitionspartner infrage kommt. (DR 160913-05)

3 Die Beispiele aus besagtem Dissertationskorpus werden mit dem Sigel „DR“ gekennzeichnet: Das Korpus besteht aus 20 Stunden Radiogesprächen aus den Morgenprogrammen von Deutschlandfunk und Deutschlandradiokultur zwischen dem 16.8.2013 und dem 23.9.2013. 
Eine skalare Interpretation wäre, dass sich der FDP-Politiker nicht unbedingt, oder nicht nur, eine größere Anzahl an Koalitionspartnern gewünscht hätte, sondern auch und vor allem einen (oder mehrere) bessere(n) Koalitionspartner.

Sobald die Interpretation skalar ist, nimmt die von nur gekennzeichnete Lösung einen niedrigen Wert in der Skala ein. Die Parallele mit der semantischpragmatischen Behandlung von frz. seulement leuchtet hier ein: Sowohl Ducrot ${ }^{4}$ (1980) als auch Culioli ${ }^{5}$ (1999a) haben in ihren jeweiligen theoretischen Rahmen nachgewiesen, dass frz. seulement und ne ... que ... die gleiche „argumentative Orientierung“ (Ducrot) aufweisen wie die Satznegation: Ganz wie ne ... pas entsprechen seulement bzw. ne ... que einer Umkehrung der diskursiven Orientierung im Vergleich zum Vorhergehenden.

Bien que la réalité objective désignée par à moitié pleine et à moitié vide soit la même, on n'emploie pas ces expressions pour amener à la même conclusion. [...] Seulement à moitié pleine a la même valeur que à moitié vide. C'est en effet une règle générale que l'addition de seulement dans un énoncé en transforme - [...] en inverse - la valeur argumentative. (Ducrot 1980: 25)

Wenn dieser vorhergehende Kotext nicht selber durch einen negativen Marker gekennzeichnet ist, sorgen seulement oder ne ... pas für eine „disphorische“ Umkehr: Die argumentative Kraft der Assertion wird deutlich geschwächt, weil das Paradigma von oben nach unten durchlaufen wird, vom stärksten bis hin zum schwächsten Argument in der Klasse. Nur schließt somit alle stärkeren Varianten aus und signalisiert, dass die argumentative Basis für die These eigentlich niedrig ist. Eine solche Paraphrase, sollte sie auch für nur gelten, wirft nun eine wichtige Frage auf, was die historische Entwicklung anbelangt.

4 Oswald Ducrot ( $\left.{ }^{\star} 1930\right)$ ist ein französischer Semantiker und Pragmatiker, der in den 1970er Jahren, allein und in Mitarbeit mit Jean-Claude Anscombre, eine Theorie der sprachlichen Argumentation entwickelt hat, in der Sprache als inferenzbasiertes Kommunikationssystem behandelt wird. Innerhalb einer Sprache seien immer eine Reihe von Formen vorhanden, die für den Ausdruck der Inferenz- und Argumentationsmechanismen spezialisiert seien. Im Wesentlichen handelt es sich um Diskursmarker. König (1991: 169ff.) beschreibt Ducrots Modell aus den 1970er und frühen 1980er Jahren als Vorläufer modernerer relevanztheoretischer Arbeiten zur Diskursmarkerforschung. Spätere Entwicklungen dieses Modells sind für uns hier nicht von Belang.

5 Antoine Culioli ( $\left.{ }^{\star} 1924\right)$ ist ein französischer Semantiker germanistischer Ausbildung. Er ist ein wichtiger Vertreter der „enunziativen“ Tradition der französischen Linguistik, die sich auf Intersubjektivität, Kontexteffekte und Diskursphänomene konzentriert. Zu den Kernthemen von Culiolis Schaffen gehören neben Diskursmarkern und Satzmodus auch Aspekt und Modalität. Der Hauptunterschied zu Forschungsansätzen wie denen von Bühler oder Coseriu im deutschsprachigen Raum, oder von Ducrot in Frankreich, ist die methodologische und theoretische Abneigung zum Begriff der Sprecherabsichten. 
Ursprünglich stand nur für eine positive Ausnahme in einem nachdrücklich negativen $\operatorname{Ko}(\mathrm{n})$ text, d.h. für eine euphorische, nicht disphorische Wende. Diese semantische Entwicklung von ni waere zu nur erfolgte jedoch parallel zum Verschwinden der Verneinungspartikel ni, die nicht nur in der Morphologie von ni waere vorkam, sondern auch die Segmente im Vorkontext als negativ kennzeichnete (siehe Bsp. (1) oben).

Wie Duplâtre (2015) in seiner Studie über die historische Entwicklung von kein nachgewiesen hat, hat dieser Wegfall in manchen Fällen dafür gesorgt, dass Negative Polarity Items, d.h. Formen, die erst im Skopus eines negativen Operators $^{6}$ vorkommen durften, nun selber für die bisher von ihnen verlangte negative Polarität sorgen konnten. Dadurch entwickelte sich kein vom indefiniten Artikel negativ-polarer Kontexte (vgl. zu engl. any) zum Nullquantor, und mithin zum Auslöser der negativen Polarität. Unsere Vermutung folgt den Prinzipien des Jespersen-Zyklus und lautet wie folgt: Der Wegfall der Negationspartikel ni hatte eine umfassende Restrukturierung des Systems der polaritätssensitiven Formen im Hochdeutschen zur Folge. Im Zuge dieser Restrukturierung haben einige Marker, darunter kein und nur, die Fähigkeit erhalten, die negative Polarität, die bisher erst von ni ausgelöst werden musste, selber auszulösen.

Ursprünglich führte der Konnektor nur eine positive Ausnahme zu einem negativen Kontext ein. Diese negative Polarität des Vorkontextes war an einem anderen, vorher realisierten Marker zu erkennen, tendenziell ni. Nach dem Wegfall von ni sorgt nur selber für diese negative Wende, und begrenzt sie zugleich, indem sie auf den unreduzierbaren Kern von Positivität auch hinweist.

Soweit, so gut? Bei näherem Betrachten der Daten erweist sich diese Argumentationsweise als allzu simpel. Mehrere Gebrauchsweisen von nur passen nur schwer in diesem Schema zusammen:

- Nur als Konnektor

- Nur als Diskurspartikel mit breitem Skopus

- Nur als nicht-exklusive Fokuspartikel

- Nur als Irrelevanzpartikel

Letztere drei Gebrauchsweisen sind historisch aus demselben Strang entstanden, wie die exklusive Fokuspartikel; der heutige Konnektor hingegen scheint sich von Anfang an von den anderen abgetrennt zu haben und stellt in vielen Hinsichten ein Relikt aus der Grundbedeutung von ni waere dar.

6 Zur Diskussion siehe Giannakidou (2011). Über das Verhältnis von Polaritätssensitivität und äußerungsbezogener Bedeutung siehe Ernst (2009). 


\section{Konvergenz und Divergenz in der semantischen Entwicklung von nur als Konnektor}

\subsection{Negation der Negation}

Fangen wir also von Neuem an. Ni waere (p) schloss sich Kontexten an, die auch formal als negativ erkennbar waren.

(8) ûz iwerm dienst in nimmer nôt/vertrîben mac, niwær der tôt. [Lichtenstein 128, 6 , zit. DWB XIII: 999]

Das negative Urteil galt ausschließlich für die Fälle, in denen die Bezugskonstituente von ni waere ausgeschlossen werden konnte. Nun ging man eben davon aus, dass diese Bezugskonstituente NICHT ausgeschlossen werden sollte. Die Irrealisform (wäre) zeigte an, dass dieser Ausschluss der Bezugskonstituente (ni) unbegründet war. Wenn wir bei Ducrot bleiben, heißt das, dass ni waere den Ausschluss aller Gegenargumente aufhebt und seine Bezugskonstituente gelten lässt. Auf das negative Urteil, das im Vorkontext ausgedrückt wurde, folgt eine positive Aussage, die das negative Urteil teilweise zurücknimmt. Was nun, wenn diese Beschränkung auf negative Vorkontexte wegfällt?

Erste Möglichkeit: Nur nimmt die Fähigkeit an, die negative Polarität selber auszulösen. Dieser Weg entspricht der semantischen Entwicklung der Fokuspartikel.

Zweite Möglichkeit: Es passiert nichts, bis eben auf die Tatsache, dass die vorherige Aussage nicht mehr negativ sein muss. Der Konnektor kann somit zwei positive Aussagen verknüpfen, so etwa im folgenden Beispiel:

(9) GAST: Denn wenn es um Sicherheit und um Freiheit geht, dann haben wir begrenzte Mittel, gerade was sozusagen auch Haushaltsmittel angeht, die müssen wir effektiv einsetzen, und da hab ich manchmal das Gefühl, dass da doch die eigene Eitelkeit mehr zählt als die Gesamtschau. Und das ist sicherlich eine sehr große Aufgabe, an der sich aber nicht nur der Bund, sondern eben auch und gerade die Länder beteiligen müssen.

JoURNALIST: Nur, wenn das passiert, ich glaube, niemand wird widersprechen, dass das passieren muss, wenn es aber passiert, dann steht doch die Frage im Raum, wie können wir das kontrollieren, denn die verschiedenen Länderbehörden werden alle nur einzeln kontrolliert. (DR 240813-05) 
Nur führt hier ein Gegenargument zur Aussage des Sprechers A ein: Die Länder mögen zwar ihre Hoheit behalten (das ist die These von A), aber so pauschal gelte diese Aussage erst in einem Kontext, in welchem es nicht der Fall wäre (ni waere!), sodass die Frage im Raum steht, wer den bundesweiten Überblick haben sollte. Die argumentative Wende erinnert also sehr stark an diejenige, die ursprünglich von ni waere markiert wurde, allerdings unter anderen polaren Voraussetzungen.

\subsection{Divergenz und Konvergenz in der Semantik von nur}

Mit anderen Worten: Während die exklusive, disphorische Fokuspartikel den Wert des gesamten argumentativen Konstrukts übernommen hat, in welchem $n i$ waere vorkam (,einzige Ausnahme zu einem negativen Urteil“), hat der Konnektor die kompositionale Semantik von ni waere in ne $\boldsymbol{q}$, ni waere $\boldsymbol{e}$ (,Die vorige Aussage nicht-q ist nur in dem Fall annehmbar, wo man die Tatsache verkennt, dass e der Fall ist“) behalten.

Konnektor1: ne q, ni waere e:

$\mathbf{e}=$ einzige Ausnahme zu einem negativen Urteil über $\mathbf{q}$

Das Bekenntnis $\mathrm{zu}<$ ne $\mathbf{q}>$ beruht auf der Ignoranz von $\mathbf{e}$.

Beide Umschreibungen sind ursprünglich äquivalent. Anders jedoch bei den zwei direktesten Erben:

Fokuspartikel: (q (nur e))

$\mathbf{e}=$ einige Ausnahme zu einem negativen Urteil über $\mathbf{q}$

Konnektor2: q, nur e

Das Bekenntnis zu q beruht auf der Ignoranz von e.

Allem Auseinanderdriften zum Trotz scheint diese konnektive Verwendung von nur im Standarddeutschen immer noch mit den anderen Gebrauchsweisen verbunden zu sein. Im Kontrast zum Niederländischen oder zum Jiddischen, wo sich maar resp. nur als „normale“ adversative Konjunktionen weiterentwickelt haben (zur Besprechung, siehe König 1991: 106f.), scheint nämlich das standarddeutsche nur Präferenzen für bestimmte Kontexte aufzuweisen, in denen eigentlich 
eine semantische Verbindung zu den übrigen Gebrauchsweisen sich durchaus etablieren lässt. ${ }^{7}$

(10) Die Berichte vom gestrigen Abend deuten ja nicht darauf hin. Auf der anderen Seite hat auch Putin gesagt, wenn es denn unmissverständliche Beweise gäbe, dann wäre er auch dabei, in Syrien energisch durchzugreifen. Nur, das setzt ja voraus, dass man dann auch die Untersuchung nicht blockiert. (DR 060913-04)

Der Konnektor nur kommt sehr oft unmittelbar nach einem Sprecherwechsel vor und wird nicht selten als ironisch interpretiert, bzw. als würde der Sprecher das Gewicht des Gegenargumentes herunterspielen. Daraus kann man folgende Hypothese ableiten: Das Vorhandensein von Gegenargumenten gilt in diesen Kontexten als präkonstruiert. Verneint wird diese Klasse von Gegenargumenten, bis auf eine einzige Ausnahme: den Gehalt des neuen Teilsatzes. Ganz im Sinne der „unvollständigen Verneinung“ findet sich dieser Konnektor oft in euphemistischen oder in vermindernden Kontexten: Die Ausnahme ist nicht in der Lage, die allgemeine Tendenz zur Zurückweisung aller Gegenargumente umzukehren. Daher eine Interpretation, in der das von nur eingeführte Gegenargument einen minimalen argumentativen Wert hat, was wiederum oft für ironiche Gebrauchsweisen sorgen kann. Diese Hypothese wurde von Modicom (2016: 356-360) vorgestellt, damals aus der Sicht des Bedeutungsminimalismus und mit dem Ziel, die Bedeutung des Konnektors in Einklang mit derjenigen der Fokuspartikel zu bringen. Die diachronen Daten und die Hinweise auf benachbarte Sprachen deuten auf einen komplexeren Zusammenhang hin. Festzustellen ist außerdem, dass frz. seulement und sauf que eine ähnliche Distribution aufweisen. Sauf que erinnert sehr stark an das ursprüngliche ni waere und hat keinen Fokuspartikelgebrauch. Seulement hingegen liefert ein schönes Beispiel der Konvergenz zwischen exklusivem Operator und adversativem Konnektor, ohne dass Polarität je eine Rolle gespielt zu haben scheint. Interessant ist zudem die Tatsache, dass die Sprachen, in denen ein Cousin von nur zur häufigsten adversativen Konjunktion geworden ist (d.h. wo der Minderungseffekt verlorengegangen ist), auch diejenigen sind, in denen dieser Cousin von nur in der fokus-sensitiven Operatorfunktion am meisten bedrängt ist, als würde die Häufigkeit des exklusiven Operator-

7 Hinzu kommen noch einige Grenzfälle, in denen nur mit einem ähnlichen „konnektiven“ Wert im Mittelfeld vorkommt: Das ist eine Art Käsegericht, warm zubereitet, überbacken, dazu verschiedene Arten von Soße, Tomatensoße, man sollte es nur nicht mit Worcestershiresauce probieren. (zit. Kwon 2005: 130). 
gebrauchs auf die Interpretation des adversativen Konnektors zurückwirken. Zwei Arten von Kausalität scheinen hier zu interagieren: die Sprachentwicklung als Faktor der semantischen Divergenz und pragmatische Regelmäßigkeiten als Faktoren der Konvergenz.

\section{Alternativensemantik mit und ohne Fokussierung}

Der Konnektor ist aber bei Weitem nicht der einzige Gebrauch von nur, für welchen der von ni waere vererbte Begriff der „unvollständigen Verneinung“ an seine Grenzen stößt.

\subsection{Fokus und Domäne}

Fangen wir an mit dem „einfachsten“ Fall, der „Diskurspartikel“ nur:

(11) Er ist jedoch der Meinung, dass nicht erst Sigmar Gabriel hätte kommen müssen, um „die Dinge zu richten“. „Man hätte nur dem einstimmigen Beschluss des Ortsrates folgen müssen, der die Verwaltung und damit das Straßenbauamt Goslar beauftragte, die alternative Lösung für eine gesonderte Baustellenzufahrt zu schaffen“, betont Nüsse. (DEREKo: Braunschweiger Zeitung, 9.9.2005)

Diese Gebrauchsweise wird manchmal dem Bereich der Modalpartikeln zugerechnet (siehe Kwon 2005; ansatzweise schon Bayer 1999). Eine solche Klassifizierung beruht auf der Annahme, dass Fokuspartikeln einen „Skopus“ entwickeln, der auf eine Konstituente beschränkt sei, während „Modalpartikeln“ (bzw. Abtönungspartikeln) die gesamte VP in ihrem Skopus umfassen würde. Diese Dichotomie ist aber inzwischen weitgehend widerlegt. Ausgehend unter anderen von König (1991) wollen wir hier zwischen dem Fokus einer Partikel und seiner Domäne unterscheiden. „Fokuspartikeln“ sind demnach Operatoren, die den semantischen Gehalt ihrer Domäne (i.d.R. die gesamte VP) in einer Alternativenmenge situieren. Sie sind aber fokussensitiv. Das heißt: Gibt es in der Domäne eine Konstituente, die etwa über einen Kontrastakzent fokussiert wird, so wird die Partikel an diese Fokuskonstituente gebunden und in Bezug auf sie interpretiert: Die Komponenten in der Alternativenmenge unterscheiden sich von der ausgewählten Lösung nur durch den Wert der Bezugskonstituente; der Rest der 
Domäne bleibt konstant. In Beispiel 7, das an dieser Stelle wiederholt wird, besteht die Alternativenmenge aus den übrigen Parlamentsparteien.

(7) Es ist leider so, das ist unser Schicksal, dass im Moment nur die CDU \{und/oder die SPD, die Grünen, die Linke, die AfD...\} für uns als Koalitionspartner infrage kommt (DR 160913-05)

Restriktive Operatoren wie nur oder additive wie auch können als „Fokuspartikeln“ fungieren, d.h. eigentlich als fokusgebundene Alternativoperatoren, sobald ein Fokus im Satz vorhanden ist. Letzteres muss aber nicht der Fall sein. Dann wird der semantische Wert des Operators auf die gesamte Domäne angewendet. Hier heißt es: Die gesamte Domäne wird als eine schwache Lösung vorgestellt, die sich aus der sukzessiven Entfernung aller höheren Lösungen im Paradigma ergibt.

\subsection{Hinreichende Bedingung}

In manchen Fällen werden die höheren Lösungen nicht als falsch ausgeschlossen, sondern als unnötig aufwendig. König (1991: 98-101) spricht hier von einem Ausdruck der hinreichenden Bedingung. $\mathrm{Zu}$ diesem Bereich gehört auch der Gebrauch in den Imperativsätzen und in den Wunschsätzen.

(12) ach sehet nur, wie hat sie so ein glatte stirn! [Grimmelshausen, zit. DWB XIII: 1006]

Mangels einer einzigen prosodisch fokussierten Konstituente wird die gesamte VP einer Menge unbestimmter Alternativen entgegengesetzt. In beiden Fällen geht es allein darum, die Lösung als einen niedrigen Punkt in der Skala der kontextuell möglichen Vorgänge vorzustellen. Im Falle des kontrafaktischen Wunschsatzes ergibt dies eine Verstärkung des illokutiven Merkmals BEDAUERN, da die nicht verwirklichte Handlung als ein doch recht bescheidener Wunsch erscheint: Nicht einmal dieses Minimum konnte oder durfte stattfinden, obwohl die Erfüllung dieses Wunsches angeblich für das Glück des Sprechers ausreichend gewesen wäre.

In Imperativsätzen markiert dieses nur eher, dass die Handlung eine Geringfügigkeit ist, oder nicht viel Kraft bzw. Mut verlangt. Illokutionsmäßig ergeben sich zwei große Lesarten: eine willkommenheißende (wenn der Adressat auf eine Erlaubnis wartet) und eine ungeduldige (wenn der Adressat nicht willens 
ist, die Handlung auszuführen). Hinzu kommt noch eine konventionalisierte Bedrohungslesart. Der Imperativsatz dient hier als Protasis einer unvollendeten Periode, deren Apodosis jedoch kontextuell eindeutig ist: „Du wirst sehen, was dir passiert“. Die Tatsache, dass der Gehalt des Imperativsatzes hier durch nur minimisiert wird, rückt diese negativen Befehle in die Nähe der Argumente $a$ fortiori und verstärkt somit die Bedrohung.

Eine weitere Form von hinreichender Bedingung, diesmal mit Fokusbindung, ist der nicht-exklusive Gebrauch in Bezug auf thematische Konstituenten.

\begin{abstract}
Das ist vollkommen richtig, wir haben ja, die Verkehrsminister der Länder haben ja eine Kommission gebildet, in der ich auch Mitglied war, und wir haben sozusagen gemeinsam festgestellt, dass es in Deutschland pro Jahr etwa ein Dezit von rund sieben Milliarden alleine beim Erhalt und bei der Sanierung von Infrastruktur, also Straße, Schiene, Wasserstraße insgesamt gibt, also nur beim, nur beim Erhalt und bei der Sanierung. (DR 170813-01)
\end{abstract}

König (1991: 99) merkte schon an, dass die Domäne der Partikel sich beim Ausdruck der hinreichenden Bedingung auf die Fokuskonstituente beschränkt (,narrow scope“). Die Phänomene in den Imperativsätzen scheinen diese Lesart teilweise zu widerlegen, da es in diesen Sätzen sehr wohl eine Domäne (in 12 etwa die ganze VP) gibt, die sich nicht auf eine Fokuskonstituente reduzieren lässt. Bemerkenswert bleibt die Tatsache, dass die nicht-exklusiven Lesarten mit einer Neutralisierung des Unterschieds zwischen Fokus und Domäne korreliert sind. Es geht nicht darum, den Wahrheitsgehalt der Domäne auf den Wert der Fokuskonstituente $\mathrm{zu}$ indizieren. Vielmehr beschäftigen sich die nicht-exklusiven Gebrauchsweisen von nur mit der argumentativen Gewichtung des Gehalts. Die Domäne von nur wird als minimal stark interpretiert - wiederum geht es um eine Bewegung nach unten. Daraus entsteht manchmal, vor allem wenn die Domäne selber eine (thematische) Konstituente einer Aussage ist, die Möglichkeit eines Arguments a fortiori wie in (13).

Ein Extremfall der hinreichenden Bedingung, und zugleich ein Grenzfall mit dem Bereich der Irrelevanzmarkierung, ist die Kombination von nur mit einem modalen Prädikat im Skopus eines Allquantors oder eines W-Worts:

(14) Es gibt kein staatliches Gewaltmonopol mehr: Alle morden, wo sie nur können. (Georg Kreisler, Schlagt sie tot !)

(15) In der Welt der deutschen Wissenschaftsfunktionäre gelten nämlich andere Maßstäbe. Das ist das eigentliche Problem, das der Fall Schavan 
offenbart hat. Von den Priestern dieser Gemeinde erfuhr die Ministerin monatelang alle nur denkbaren Segnungen. ${ }^{8}$ (FAZ, 9.2.2013)

Im ersten Fall reicht jede beliebige durch wo dargestellte Situation, damit die Voraussetzungen für den durch morden ausgedrückten Vorgang geschaffen werden. Im zweiten Fall kommt die hinreichende Bedingung noch deutlicher zum Vorschein: Man braucht nur an irgendeine beliebige Segnung zu denken, um der im Satz dargestellten Lage gerecht zu werden. Die modalen Prädikate werden hier als Minimalwerte in ihren jeweiligen Skalen benutzt.

\subsection{Irrelevanz}

Dennoch ist diese Konstruktion nicht allein mit modalen Prädikaten möglich.

$$
\text { Wer es nur nam, dem gab er. [Aventinus ( 1500), zit. DWB XIII: 1007] }
$$

Sie kann außerdem nur von einem Allquantor oder $W$-Wort ausgelöst werden, und nicht etwa von einem unspezifischen Indefinit, wie das für auch nur möglich ist.

(17) Um die Annahme von Verschörtungstheorien und -Praxen der Männer oder einer Theorie weiblicher Subjektwerdung unter Verzicht auf eine auch nur denkbare Beteiligung der Frauen an der Macht zu vermeiden, möchte ich probeweise versuchen, Frauenunterdrückung und die Frauenfrage an einen Ort zu knüpfen [....] (Frigga Haug 1984, Internetbeleg) ${ }^{9}$

Und in Fragen:

Wo soll ich nur hingehen?

Beide Gebräuche teilen einen semantischen Wert, der in der formalen Literatur widening ('Erweiterung') genannt wird und mit dem Begriff Free Choice zusammenhängt (Chierchia 2006). Der Widening-Effekt, der im Deutschen v.a. mit

8 Man vergleiche etwa mit Vielleicht wollte sie mich daran erinnern, dass diese Gesetzmäßigkeit nur eine scheinbare war. (Julia Schoch, Mit der Geschwindigkeit des Sommers, 2009).

9 Aus Bergen, Monika (Hg.) (1984): Karriere oder Kochtopf? Frauen zwischen Beruf und Familie. Hamburg: Westdeutscher Verlag. 24. 
immer und/oder auch markiert wird (Leuschner 2000), manifestiert sich etwa in der Veränderung eines $W$-Worts in einen distributiven Allquantor, mit welchem eine referenzielle Klasse vollständig durchlaufen wird, d.h. inklusive der normalerweise pragmatisch ausgeschlossenen Extremfälle. In nicht-assertiven Kontexten sorgt das für eine radikale Zurückweisung aller denkbaren Lösungen, in assertiven Kontexten hingegen sorgt die free choice für die Inklusion und Validierung sämtlicher möglichen Lösungen; daher die unfesten Grenzen mit dem Ausdruck der hinreichenden Bedingung im Falle von alle nur denkbaren Segnungen oder wo sie nur können. Interessant ist hier zudem die diskursive Äquivalenz zwischen nur und auch (zum Kommentar, siehe Modicom 2016: 380f.).

Der Schlüssel hier ist der distributive Allquantor. In seiner Analyse von frz. Qu- erklärte Culioli (1999c), dass das Fragewort eine gesamte Klasse bedeutet, die vollständig durchlaufen wird, ohne dass eine Lösung aus der Menge ragt. Steht der Operator im Skopus eines positiven Operators (etwa dem der Assertion in der formalsemantischen Satzmodusforschung), so bildet die Klasse selbst die Lösung. Steht der Operator im Skopus eines nicht-assertiven Operators (Frageoperator, Konditionaloperator ...), so endet der Durchlauf ohne Ergebnis, und das endgültige Urteil wird einem anderen Sprecher überlassen. Nur liefert sehr gute Argumente für diese Analyse. Eine diskriminierende Lesart von nur ist unverträglich mit der Verbindung zu einem Allquantor, da dies implizieren würde, dass man etwas über dieses „Alle“ hinaus denken würde, was ja widersprüchlich wäre. Hier wird also die Fokuskonstituente nicht einer anderen Lösung im Paradigma entgegengesetzt: Sie ist das Paradigma. Bei allem Unterschied muss festgestellt werden, dass diese Lesart, da sie im Grunde immer inklusiv ist, semantisch und wohl auch historisch gesehen auf ebendiese hinreichende Bedingung zurückzuführen ist.

Der Widening-Effekt besteht also in der Inklusion von Randwerten. Die Partikeln auch und nur spielen hier eine wichtige Rolle: Sie verdeutlichen die Durchlaufoperation, indem sie ihre Richtung angeben (auch: nach oben; nur: nach unten). Auch markiert, dass „hoch liegende“, primär außer Acht gelassene Lösungen in Betracht gezogen werden. ${ }^{10}$ Dasselbe gilt für immer (je-mehr). Nur hingegen schließt niedrige Werte ein, weshalb diese Konstruktion gerne mit

10 Problematisch ist, dass es sich dabei vielleicht nicht um ein addierendes auch handelt. Wie Duplâtre (2006) gezeigt hat, kann das konzessive auch vom hinzufügenden auch unterschieden werden. Zwar mag man spontan an das „Addieren aller nur erdenklichen Möglichkeiten“ denken. Die Tatsache aber, dass der Sprecher durch den Gebrauch von auch das Durchlaufen des Paradigmas einem anderen Sprecher überlässt, plädiert dafür, dass dieses auch an konzessive Kontexte gebunden ist. 
nicht-faktischen Prädikaten verbunden ist, oder mit einfachen Wahrnehmungsverben, die noch keine Handlung voraussetzen. Nur markiert, dass die Klasse nach unten durchlaufen wird, dass sämtliche Lösungen getrennt in Betracht gezogen werden (daher der Allquantor), und dass der Wahrheitswert auch für die geringsten Lösungen gilt, was für die pragmatische Nähe mit der ausreichenden Bedingung sorgt.

Wir kommen nun zu den Fällen, wo nur in Kombination mit so zum Ausdruck des starken Grades beiträgt:

Das zyklopische Auge, das uns den Eindruck allseitiger und immerwährender Beobachtung unterhalb der Bewußtseinsebene vermittelt, das Auge von »Big Brother « also, gehört keinem menschlichen »Inspektor« an, obwohl es mehr denn je von »Inspektoren« nur so wimmelt. (zit. DWDSKernkorpus)

Auch diese Fälle könnten zwar zum ausschließenden nur gerechnet werden. Das Vorhandensein eines den hohen Grad ausdrückenden Wortes im Skopus von nur ist jedoch mit dem Begriff der Exklusivität im Signifikat von nur schwer zu vereinbaren. Solche Gebräuche lassen sich daher kaum in eine Systematik der Varianz von nur einschließen. Es sei denn, man könnte diese Gebräuche in die Nähe des Irrelevanz-nur rücken. Von Belang wäre hier der Durchlauf einer vollständigen Skala qualitativer Natur (etwa einer Skala von Graden?). Nur würde sich mit einem Morphem kombinieren, das ähnlich wie $W$ - entweder indefinit ist, oder den gesamten Umfang einer Klasse bedeutet. Im Fall von so ist diese vage Bedeutung schon spezialisiert für den Ausdruck der Intensität, nach einem Modell, das in Culioli (1999b) reichlich beschrieben wird. So weist auf keinen festgelegten Wert hin, sondern auf einen qualitativen Skalendurchlauf, der wiederum in einen Punkt mündet, welcher zugleich archetypisch und extrem ist (Culioli nennt diesen Wert le centre attracteur, das Anziehungszentrum oder l'attracteur, den Anzieher). ${ }^{11}$

Considérons maintenant, de façon spécifique, le marqueur si, par exemple dans si chaud. Ce marqueur indique la construction d'une relation entre deux occurrences d'une certaine propriété (p.ex. chaud, dans si chaud). Cette relation en boucle se fait sur un gradient (de non-chaud à de plus en plus chaud, jusqu'à vraiment chaud); la boucle est donc [...] nécessairement orientée vers l'attracteur. Mais l'attracteur fournit une valeur limite, qui ne peut être atteinte. On obtient ainsi une valeur instable [...]. On peut montrer de façon indépendante

11 Dieses Modell gilt auch im Falle einer anaphorischen Lesart von so oder si. Zum Zusammenhang zwischen Anapher und Intensivierung siehe noch Culioli (1999c: 132). 
qu'une valeur instable doit trouver une issue qui la stabilise. Une première issue est fournie par l'attracteur. (Culioli 1999b: 109) ${ }^{12}$

Ob $W$-, jed- oder so: Die Fokuspartikel nur bindet sich zu einer Konstituente, die nicht für einen Wert innerhalb einer Skala steht, sondern für den Durchlauf dieser Skala, was einen Widening-Effekt auslöst, der in der Regel als Intensivierungsstrategie interpretiert wird.

\section{Schluss: Systematik der Varianz von nur}

Was ist nun mit der semantischen Einheit in dieser Vielfalt? Es gäbe zahlreiche Modellierungsmöglichkeiten, um die verschiedenen Werte von nur in der Synchronie unter ein Dach zu bringen, und/oder um die Parameter ihrer Varianz zu verdeutlichen. Wir haben uns für ein sog. Trimorph entschieden, ein Muster, das von Martine Dalmas' Kollegen Bernard Pottier an der Universität Paris-Sorbonne entworfen wurde (siehe etwa Pottier 2000). Nach diesem Modell können semantische Subsysteme geordnet werden, indem die verschiedenen Marker, oder die verschiedenen Werte eines Markers, an einer Linie entlang verteilt werden, die vom Verhältnis dreier Punkte bestimmt wird, in unserem Falle p, p (nicht-p) und nicht-p.

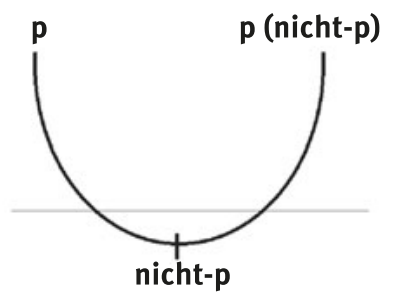

Abb. 1: Modell des Trimorph, bearb. nach Pottier (2000)

12 „Betrachten wir dann wir also nun den Marker si (,so“), etwa in si chaud (,so heiß“). Dieser Marker signalisiert, dass zwei Okkurrenzen einer bestimmten Eigenschaft (etwa chaud, „heiß“, in si chaud) in Verbindung gesetzt werden. Diese Verbindung nimmt die Form einer Schleife und erfolgt auf einer Skala (von non-chaud, „nicht-heiß“, bis hin zu vraiment chaud, „wirklich heiß“, über de plus en plus chaud, ,immer heißer“). Die Schleife orientiert sich daher zwangsläufig auf das Anziehungszentrum. Das Anziehungszentrum stellt aber einen Grenzwert dar, der nicht erreicht werden kann. Der Wert bleibt also nicht-stabilisiert. [...]. Unabhängig davon kann man zeigen, dass ein unstabiler Wert einen stabilisierenden Ausgang finden muss. Eine erste Ausgangsmöglichkeit ist das Anziehungszentrum selber.“(Culioli 1999b: 109). 
Der erste Pol, p, kennt noch keinen Unterschied zwischen dem Behaupteten (p) und seiner Verneinung (nicht-p). Das Irrelevanz-Nur böte sich hier an: Es kennt keine Exklusivität und seine Polarität hängt völlig vom syntaktischen Kontext ab. Der Gegenpol, p (nicht-p), ist die völlige Bejahung von p im Gegensatz zu einem präkonstruierten nicht-p. Der heutige Konnektor gilt hier als idealer Kandidat. Dazwischen gibt es eine Bewegung hin zu und weg von einem Nullpunkt (der Behauptung von nicht-p), also von p zu nicht-p und von nicht-p zu p. Die Eigenschaft dieses Modells, im Vergleich etwa zu seiner Inspirationsquelle, dem Tenseur binaire von Guillaume (siehe Guillaume 1946/1985: 63f. sowie Guillaume/ Valin 1973: 200f.), besteht darin, dass der Tiefpunkt (Behauptung von nicht-p) nie vollständig erreicht wird, sodass der untere Teil des Trimorphs von einer waagerechten Linie abgesondert wird, unter welcher eine „Grauzone“ liegt, der jedoch eine strukturierende Rolle zufällt. Nach diesen Prämissen bekommen wir folgendes Schema:

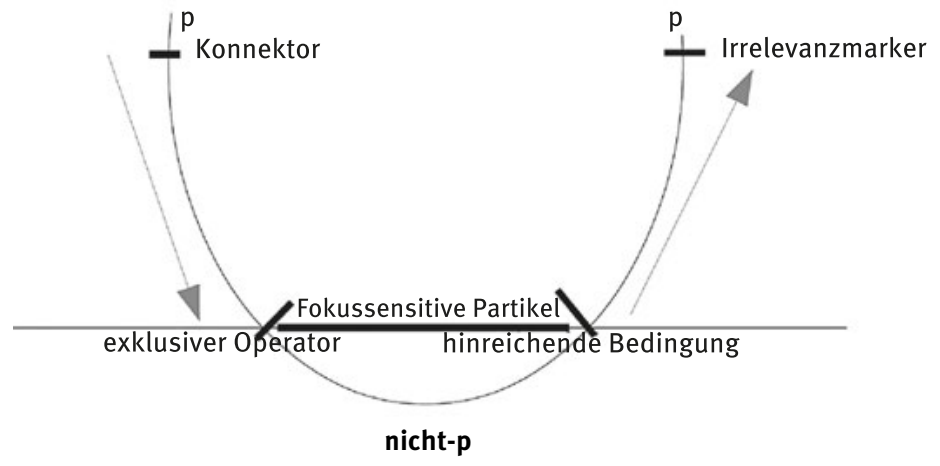

Abb. 2: Systematische Verteilung der Verwendungen von nur im heutigen Deutsch

Dazu mehrere Kommentare:

Die notwendige und die hinreichende Bedingung stehen einander gegenüber, und stehen in direkter Verbindung. Das Modell isoliert die negierbaren Gebrauchsweisen von nur, die der waagerechten Linie (ausschließlich der hinreichenden Bedingung) entsprechen.

Die Grauzone steht für den Bereich, wo der Gehalt der Assertion ein „nichtp“ ist, und wo die Behauptung von p nur remanent bleibt. Zwar wird der absolute Nullpunkt nicht erreicht, bzw. er würde von der einfachen Satzverneinung ausgedrückt werden. Bemerkenswert ist aber die Tatsache, dass dieser semantische 
Bereich (Dominanz von nicht-p, negative Polarität, Remanenz von p) den ursprünglichen, verlorengegangenen Gebrauchsbedingungen von ni waere (,es sei denn“) entspricht.

Die Modellierung durch das Trimorph ist somit weitgehend äquivalent zur Darstellung der historischen Verbindung zwischen den verschiedenen Gebrauchsweisen von nur:

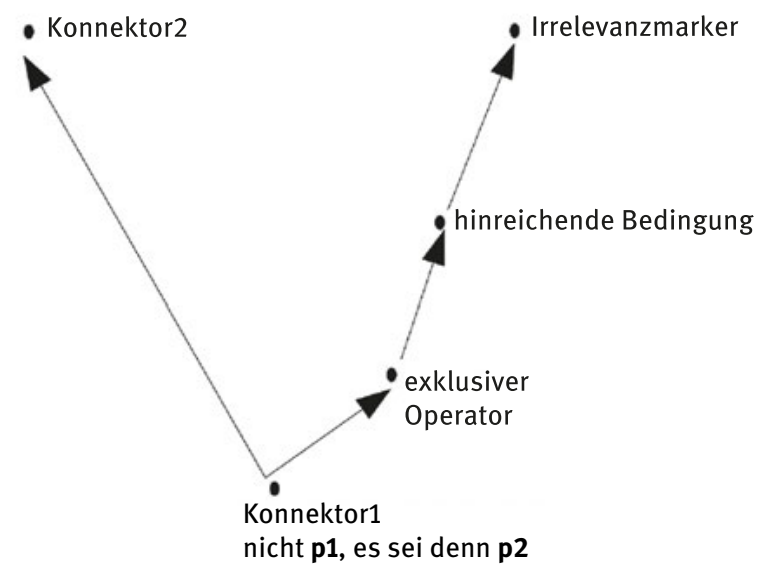

Abb. 3: Historische Entwicklung der Gebrauchsweisen von nur

Soweit für die Divergenz. Dieses Schema soll aber nicht vergessen lassen, dass es im Gebrauch von nur deutliche Konvergenzerscheinungen gibt, allen voran zwischen dem Konnektor und der Fokuspartikel (siehe oben). Dies geht so weit, dass einige Fälle für den um Klassifizierungen besorgten Linguisten unentscheidbar bleiben. Prototypisch dafür sind Wendungen wie das Problem ist nur, dass $\boldsymbol{p}$, in welchen nur einerseits die Gruppe [dass p] skalar-exklusiv kommentiert, andererseits aber den für den heutigen Konnektor typischen Wert des minimalen Gegenarguments aufweist, etwa in dem (selbstverständlich frei erfundenen) Beispiel 20:

(20) Semantische Klassifizierungen sind schön. Das Problem ist nur, dass man sie vielleicht zu schnell verabsolutiert.

Von letztgenannter Schwäche freilich ist die Jubilarin immer frei geblieben. Diese Vorsicht soll auch für den vorliegenden Beitrag gelten. 


\section{Literatur}

Badiou-Monferran, Claire/Buchi, Eva (2012): Plaidoyer pour la désolidarisation des notions de pragmaticalisation et de grammaticalisation. In: Neveu, Franck et al. (Hgg.):

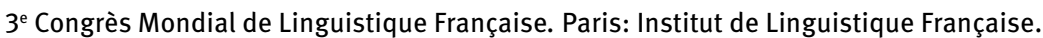
127-144.

Bayer, Josef (1999): Bound focus or how can association with focus be achieved without going semantically astray? In: Rebuschi, Georges/Tuller, Laurice (Hgg.): The grammar of focus. Amsterdam: Benjamins. 55-82.

Chierchia, Gennaro (2006): Broaden your views. In: Linguistic Inquiry 37. 535-590.

Culioli, Antoine (1999a): À propos de la notion. In: Pour une linguistique de l'énonciation 3. Gap: Ophrys. 17-33.

Culioli, Antoine (1999b): Un si gentil jeune homme! Et autres énoncés. In: Pour une linguistique de l'énonciation 3. Gap: Ophrys. 101-111.

Culioli, Antoine (1999c): Quantité et qualité dans l'énoncé exclamatif. In: Pour une linguistique de l'énonciation 3. Gap: Ophrys. 125-134.

Dostie, Gaétane (2004): Pragmaticalisation et marqueurs discursifs. Analyse sémantique et traitement lexicographique. Brüssel: de Boeck/Duculot.

Ducrot, Oswald (1980): Les échelles argumentatives. Paris: Minuit.

Duplâtre, Olivier (2006): Auch - eine semantische Untersuchung. In: Sprachwissenschaft 31. 73-111.

Duplâtre, Olivier (2015). Kein: Vom Indefinitum zur Negation. In: Pasques, Delphine (Hg.): Komplexität und Emergenz in der deutschen Syntax. Berlin: Weidler. 241-257.

Ernst, Thomas (2009): Speaker-oriented adverbs. In: Natural Language and Linguistic Theory 27. 497-544.

Giannakidou, Anastasia (2011): Positive polarity items and negative polarity items: variation, licensing, and compositionality. In: Maienborn, Claudia/Heusinger, Klaus von/Portner, Paul (Hgg.): Semantics: An International Handbook of Natural Language Meaning. Berlin: De Gruyter. 1660-1712.

Guillaume, Gustave (1946/1985): Leçons de linguistique de Gustave Guillaume, 1945-1946, série C, Grammaire particulière du français et grammaire générale I. Québec: Presses de l'Université Laval/Lille: Presses Universitaires de Lille.

Guillaume, Gustave/Valin, Roch (1973): Principes de linguistique théorique de Gustave Guillaume. Québec: Presses de l'Université Laval/Paris: Klincksieck.

König, Ekkehard (1991): The meaning of focus particles. A comparative perspective. London: Routledge.

Kwon, Min-Jae (2005): Modalpartikeln und Satzmodus: Untersuchngen zur Syntax, Semantik und Pragmatik der deutschen Modalpartikeln. Dissertation der LMU München.

Lehmann, Christian (2015): Thoughts on grammaticalization. Berlin: Language Science Press. [Erstdruck 1982].

Leuschner, Torsten (2000): Wo immer es mir begegnet - wo es auch sei: Zur Distribution von Irrelevanzpartikeln in Nebensätzen mit w-auch immer. In: Deutsche Sprache 28. 342-356.

Meillet, Antoine (1912): L'évolution des formes grammaticales. In: Scientia: rivista internazionale di sintesi scientifica 12. 384-400.

Modicom, Pierre-Yves (2016): L'énoncé et son double. Recherches sur le marquage de l'altérité énonciative en allemand. Paris: Universität Paris-Sorbonne. [Dissertation]. 
Posner, Roland (1979): Bedeutungsmaximalismus und Bedeutungsminimalismus in der Beschreibung von Satzverknüpfern. In: Weydt, Harald (Hg.): Die Partikeln der deutschen Sprache. Berlin: De Gruyter. 378-394.

Pottier, Bernard (2000): Représentations mentales et catégorisations linguistiques. Louvain: Peeters.

Traugott, Elizabeth Closs (1989): On the rise of epistemic meaning in English: an example of subjectification in semantic change. In: Language 65. 31-55. 
